\title{
Development and Validation of A Guideline on Sexual and Reproductive Health of Breast Cancer Survivors in Iran: A Mixed Methods Study Protocol
}

\section{Solmaz Roshandel}

Department of Reproductive Health and Midwifery, Faculty of Medical Sciences, Tarbiat Modares University, Tehran, Iran

\section{Minoor Lamyian ( $\boldsymbol{\nabla}$ Lamyianm@modares.ac.ir)}

Tarbiat Modares University Faculty of Medical Sciences https://orcid.org/0000-0001-7033-5605

\section{Seyed Ali Azin}

Reproductive Biotechnology Research Center, Avicenna Research Institute, ACECR, Tehran, Iran

\section{Shahpar Haghighat}

Institute of Cancer Research Division of Breast Cancer Research

\section{Eesa Mohammadi}

Department of Nursing, Faculty of Medical Sciences, Tarbiat Modares University, Tehran, Iran

\section{Study protocol}

Keywords: Sexual and Reproductive Health, Breast Cancer, Clinical Practice Guideline, Mixed Methods Study, Study Protocol

Posted Date: November 4th, 2020

DOl: https://doi.org/10.21203/rs.3.rs-99725/v1

License: (c) (i) This work is licensed under a Creative Commons Attribution 4.0 International License. Read Full License

Version of Record: A version of this preprint was published at Health Research Policy and Systems on May 31st, 2021. See the published version at https://doi.org/10.1186/s12961-021-00738-6. 


\section{Abstract}

Background: Sexual and reproductive health problems significantly decrease quality of life in survivors of breast cancer. The best approach is to provide services according to evidence based guidelines developed based on their practical context. Here, we aim to develop and validate a guideline on the sexual and reproductive health of breast cancer survivors in Iran.

Methods: The guideline will be developed and validated using an exploratory sequential mixed methods approach in four phases: 1) describing sexual and reproductive health needs of survivors of breast cancer in Iran and the health services they receive in this regard 2) performing a systematic review of existing guidelines, resources, and documents on the sexual and reproductive health of breast cancer survivors worldwide 3 ) developing a guideline on the sexual and reproductive health of women who survived breast cancer in Iran based on the results of phase one and two 4) validating the guideline developed in phase three with experts and service providers in this field.

Discussion: A comprehensive and practical guideline on the sexual and reproductive health of breast cancer survivors in Iran will be developed which will be compatible with their specific needs and culture considering limited resources available. This guideline can significantly improve the quality of life in breast cancer survivors in Iran. In addition, the approach we will use here can be utilized to develop guidelines on sexual and reproductive health of female cancer survivors in general.

\section{Plain English Summary}

Sexual and reproductive health problems decrease quality of life in survivors of breast cancer. Here, we aim to develop and validate a guideline on the sexual and reproductive health of breast cancer survivors in Iran. The guideline will be developed and validated in four phases. First, we will interview women who survived breast cancer, their husbands, and health care providers asking about their sexual and reproductive health problems and needs, and the health services they have received. Then, we will review existing guidelines, documents and resources in this regard worldwide. Subsequently, we will develop the guideline based on the results of phase one and two. We will use some recommendations from existing guidelines as they are, modify some to meet the needs of Iranian patients and introduce new recommendations as required. Finally, we will discuss this with the experts and service providers in the field to validate and finalize the guideline. This guideline can significantly improve the quality of life in survivors of breast cancer in Iran. It could also be a helpful framework to develop guidelines on sexual and reproductive health of female cancer survivors in general.

\section{Background}

Breast cancer is the most common cancer in women in most countries around the world [1]. In Iran, breast cancer is not only the most common cancer in women, but also the most common cancer in the whole population regardless of their sex [2]. Patients are mostly diagnosed when they are in their forties or 
fifties with the mean age at diagnosis being 49 years. The age at diagnosis is approximately ten years younger than the global average [3]. The number of breast cancer survivors is also increasing due to early diagnoses and advanced treatments. Five-year survival rate is greater than $90 \%$ in the United States [4] and is $72 \%$ in Iran [5].

Breast cancer survivors experience sexual and reproductive health problems $[4,6]$. After Breast cancer diagnosis and treatment, all aspects of sexual function including libido, arousal, and orgasm are often deteriorated. Patients experience dyspareunia and negative mental experiences such as negative body image, loss of femininity, concerns about infertility, lack of sexual attractiveness, depression, and anxiety leading to reduced sexual satisfaction and finally discontinuing sexual relations [6, 7]. Similarly, a study in Iran showed that sexual dysfunction is more common in breast cancer survivors compared to healthy women. They experience dyspareunia and problems in sexual desire, vaginal lubrication, arousal and orgasm [8]. Sexual function also deteriorates over time in many patients. Young age, hormone therapy and poor sexual function at the time of diagnosis are the main factors associated with worse outcome [9]. Often, sexual health is not the priority. Both patients and therapists are hesitant to talk about sexual problems due to feeling embarrassed and lack of privacy, time, or required skills $[10,11]$.

In addition, many cancer treatments can cause infertility or premature menopause [12]. Infertility can be very stressful especially in women with no children [13]. Although there are currently different solutions to preserve fertility, they may not be as practical [12]. Discussing concerns about infertility with therapists can reduce patients' levels of stress, educate them about available options, and help them to decide how to preserve their fertility [14]. On the other hand, due to teratogenic effects of cancer treatments, contraception should be considered during therapy, and young patients should receive required information immediately after diagnosis $[15,16]$.

Valid clinical guidelines are required to provide appropriate evidence-based care. Clinical guidelines should carefully be developed through an explicit process considering scientific evidence, health and medical care providers' experiences, and patients' needs. These guidelines can improve making complex medical decisions by both physicians and patients, leading to higher quality of treatment and better outcomes [17]. Clinical guidelines should also be developed according to the context in which they are supposed to be used. Developing a new guideline requires access to extensive resources. To save resources and to avoid repetitions, a combination of different approaches should be utilized including adopting recommendations from existing guidelines as they are, adapting them by making changes in existing recommendations considering local circumstances, or providing new evidence-based recommendations if required [18].

Globally, a few guidelines are available for cancer survivors' care, although they may not be specific to breast cancer or sexual and reproductive health. American Cancer Society (ACS) and American Society of Clinical Oncology (ASCO) published "ACS/ASCO Breast Cancer Survivorship Care Guideline" in 2015. Some parts of this guideline address sexual and reproductive health such as body image, sexual function, infertility, premature menopause, and hot flashes [19]. European School of Oncology (ESO) and 
European Society for Medical Oncologists (ESMO) also developed a guideline for young women with breast cancer updated in 2018 entitled "ESO-ESMO 4rd International Consensus Guidelines for Breast Cancer in Young Women (BCY4)". This guideline also includes sexual and reproductive health [15]. Cancer Care Ontario (CCO) published another guideline in 2016, "Interventions to address sexual problems in people with cancer", including recommendations for all adult male and female survivors of various cancers and their sexual partners. Parts of this guideline is specific to women with breast cancer [20]. In 2016, National Comprehensive Cancer Network (NCCN) also published a guideline entitled "Sexual Function in Cancer Survivors" addressing issues related to sexual health of survivors of various cancers [21]. The ASCO also has a guideline entitled "Fertility Preservation in Patients with Cancer" regarding preserving fertility in cancer survivors including breast cancer [22]. However, to our knowledge, there has been no guideline in Iran for sexual and reproductive health of cancer survivors in general or breast cancer to be specific. A comprehensive guideline based on scientific evidence considering local and cultural circumstances can significantly improve quality of life in these patients.

\section{AIM}

Here, we aim to develop and validate a comprehensive guideline for sexual and reproductive health of breast cancer survivors in Iran taking into account local and cultural needs and circumstances.

\section{Methods}

\section{Study design:}

We will use an exploratory sequential mixed methods approach starting with a qualitative data collection and analysis, followed by a quantitative data collection and analysis, and finally combining data from both. The study will include four phases.

In phase 1, we will collect qualitative data to explore breast cancer survivors' sexual and reproductive health needs and related health services they receive in Iran. Data will be collected through in-depth semistructured interviews with women who survived breast cancer, their husbands, and also health and medical service providers. We will use purposeful sampling to maximize diversity. Sampling will continue until data saturation. Data will be analyzed simultaneously using a conventional qualitative content analysis approach.

In phase 2, we will conduct a systematic review of all available guidelines, resources and documents on sexual and reproductive health of breast cancer or other cancers survivors worldwide.

In phase 3, the authors will draft the guideline using the results from phase 1 and 2 . Subsequently, the draft will be circulated among a number of experts and service providers in the field. The guideline will be modified based on their suggestions and recommendations, finally, a meeting will be held with the panel of experts. We will use the nominal group technique (NGT) [23] to discuss the disagreements and finalize the guideline. 
In phase 4, we will collect and analyze quantitative data to validate the guideline developed in phase three. This phase includes two steps. First, the Appraisal of Guidelines for Research \& Evaluation II (AGREE II) Instrument [24] will be used to evaluate the guideline by the research team, experts from the nominal group and two experts outside the nominal group. In the next step, to assess the feasibility and applicability of the guideline, we will circulate it among a number of service Providers. We will evaluate their level of agreement with the recommendations. Their written suggestions will also be collected and integrated in the final version of the guideline.

This study was approved by the ethics committee in Tarbiat Modares University, Tehran, Iran (IR. MODARES. REC. 1397.207). The details of each phase are presented in the next section.

\section{Phase 1: Qualitative study}

\section{Participants:}

The participants will include three groups: breast cancer survivors (women) at different stages of the disease (diagnosis, treatment and follow-up), their husbands, and service providers with different levels of expertise.

We will use purposeful sampling for recruiting breast cancer survivors to maximize diversity in terms of age (peri or post-menopause), marital status (single, married, widowed or divorced), cancer stage, disease duration, and course of disease (diagnosis to follow-up). All participants (women with breast cancer and their husbands) should be willing to participate in the study, have Iranian nationality, can speak Farsi, and will be able to communicate to share their experiences.

The service providers will be selected from a wide range of people providing health and medical services to women with breast cancer including surgeons, oncologists, radiotherapists, nurses, midwives, obstetricians, infertility specialists, reproductive health specialists, counselors, psychologists, psychiatrists, follow-up personnel and policy makers. They should have at least two years of related work experience in public, private or semi-private sectors.

\section{Study setting:}

Participants will be recruited from the public, semi-private and private centers providing services to patients with breast cancer in Tehran. The time and place of the interview will be arranged, considering the participants' choices.

\section{Data collection:}

The code of ethics will be reviewed and required permissions will be obtained in advance at each center. Eligible subjects will be informed about the research objectives and ethical considerations including confidentiality, no interference with the services they receive, destroying their audio files after completing the research, anonymity of the reports, and reflecting their views with honesty and no censorship. 
Subsequently, written informed consent will be obtained to record the interview and arrangement will be made in terms of time and place of the interview. In case the participant does not consent to record the interview, only notes will be taken.

The interviews with patients and service providers will be conducted by SR (PhD candidate, female). The interviews with husbands will be conducted by one of the male physicians within the study team (SAA) since Iranian men are more comfortable sharing their views with men due to Persian culture. The interviews will be semi-structured and expanded based on the participants' replies to the initial general questions. General questions for breast cancer survivors will include: what sexual and reproductive health problems they have experienced after breast cancer diagnosis, what concerns them in this regard, if they have shared their concerns with anybody, and what health and medical services they have received. General questions for husbands will include: what has changed in their marriage and sexual life after their wife was diagnosed with breast cancer, how they have tried to solve the problems, and what health and medical services they have received. General questions for service providers will include: what sexual and reproductive health problems breast cancer survivors have, and what kind of health and medical services they receive in this regard. The audio files will be processed and analyzed as soon as possible following the interview. The interviews will continue until data saturation when no new information or codes is recorded.

\section{Data analysis:}

The data will be analyzed using the Graneheim \& Lundman (2004) method. In this method, the entire interview is considered as the unit of analysis. Meaning units are words, sentences or paragraphs containing related aspects. The meaning units are then condensed based on their manifest (a description close to the text) or latent (an interpretation of the underlying meaning) contents. Subsequently, the condensed text is abstracted, and codes, categories and themes are created on varying levels [25].

\section{Establishing rigor and trustworthiness:}

We will ensure that the data is trustworthy described by credibility, dependability, confirmability, transferability and authenticity [26]. We will use different strategies to achieve credibility including prolonged engagement, member checking and maximum diversity sampling. We will spend sufficient time for each interview and also for processing them. The processed text from some interviews will be returned to the participants to evaluate their consistency. In addition, as mentioned above, we will use a purposeful sampling technique to maximize the diversity and capture a wide range of perspectives. Data collection will continue until data saturation, and we will use multiple methods to collect the data including interviews, field notes, and investigating all documents to increase the validity of the data. To achieve confirmability, we will present all interviews and extracted codes and themes to the experts within the study group as well as experts outside the study group who are interested in this field for further investigation and confirmation. To increase dependability, in addition to member checking, everything will be well documented and will be accessible. To increase transferability, a clear and detailed description of the context, participant selection process and characteristics, and data collection and analysis will be 
provided so that other researchers can decide if the findings can be transferred to their own settings. Constant observation, prolonged engagement, field notes, and detailed and comprehensive reporting will maximize authenticity.

\section{Phase 2: Systematic review of available guidelines worldwide}

SR and one other member of the study group will independently conduct literature review to find all relevant guidelines, documents and published papers in English or Farsi since Jan 1, 2000 using general search engines (e.g. Google); databases such as PubMed (https://pubmed.ncbi.nlm.nih.gov/), Cochrane Library (https://www.cochranelibrary.com/), ScienceDirect (https://www.sciencedirect.com/), Scopus (https://www.scopus.com/home.uri), Web of Science (www.webofknowledge.com), ProQuest (www.proquest.com), CINAHL (www.ebscohost.com), Trip Database (https://www.tripdatabase.com/), and Up ToDate (https://www.uptodate.com/); specific websites regarding clinical guidelines including Guidelines International Network (G-I-N) (www.g-i-n.net), National Institute for Clinical Excellence (NICE) (www.nice.org.uk/Guidance/), ClinicalKey (www.clinicalkey.com), Scottish Intercollegiate Guidelines Network (SIGN) (https://www.sign.ac.uk/), Agency for Healthcare Research and Quality (AHRQ) (www.ahrq.gov); internationally recognized cancer related websites such as ASCO (https://www.asco.org/), NCCN (www.nccn.org) and CCO (https://www.cancercareontario.ca); and Iranian databases such as SID (https://www.sid.ir), Irandoc (https://irandoc.ac.ir/), Magiran (https://www.magiran.com/) and Civilica (https://en.civilica.com/).

The following strategies will be used to search in PubMed and will be modified for searching in the other websites: (reproductive health[MeSH Terms]) OR (sexual health[MeSH Terms])) AND (cancer[MeSH Terms]) AND (guideline[MeSH Terms]) as well as (breast cancer[MeSH Terms]) AND (guideline[MeSH Terms]).

Subsequently, recommendations related to the study objectives will be selected and categorized into groups with regards to the supporting scientific evidence behind them. Grading of Recommendations Assessment, Development and Evaluation (GRADE) framework will be used to evaluate the recommendations [27].

\section{Phase 3: Guideline development}

First, we will use PIPOH technique to develop a set of key questions[28]. This technique includes five items: Population concerned (survivors of breast cancer in Iran), Interventions of interest (sexual and reproductive health services), target Professionals (service providers at different levels), expected Outcomes (better sexual and reproductive health services), and Health care setting and context (all public, semi-private and private centers providing services to breast cancer survivors including health care centers, offices, clinics and hospitals).

Next, the study team members MR (health education expert), SAA (sexual health specialist), SH (epidemiologist), EM (methodologist) and SR (reproductive health PhD candidate) will use the findings 
from phase 1 and 2 to develop the guideline. Three techniques will be used: adoption (accepting existing recommendations from available guidelines as they are), adaptation (changing existing recommendations from available guidelines to meet local and cultural needs) and de novo development of recommendations if required [18]. Recommendations with more supporting evidence and compatibility with local and cultural circumstances will be prioritized.

After developing the initial guideline, we will use multiple techniques to ensure that the experts in the field all agree with the recommendations especially where evidence is not sufficient [23]. The guideline including recommendations and their supporting evidence will be sent to some experts in the field. We will ask them to give us their feedback based on their personal experience in written format. The study members will then investigate all comments and suggestions. Subsequently, a meeting will be held with a panel of experts. We will use NGT to discuss the items in the meeting. The recommendations with more than $80 \%$ agreement will be included in the guideline. For recommendations with disagreement, the study group will decide and this will be mentioned in the guideline. In this meeting, decision will also be made about how and when the guideline will be updated.

\section{Phase 4: Guideline validation}

This phase will include two quantitative steps.

\section{Evaluating quality of the guideline from developers' and experts' perspective:}

To evaluate the guideline quality, we will use AGREE II instrument to address its methodological rigor and transparency. AGREE II contains 23 items in 6 domains: scope and purpose, stakeholder involvement, rigor of development, clarity of presentation, applicability, and editorial independence. Each items are rated in a 7-point scale: from 1 strongly disagree to 7 strongly agree. Under each item, there is a space for further comments. A score is calculated for each of the six domains independently and should not be accrued into a single score. Domain scores are useful to evaluate the quality of the guidelines but there is no cutoff to classify high and poor quality guidelines. At the end, individuals will evaluate the guideline overall and will be asked whether they would recommend it or not. It is recommended that at least two and preferably four people access the guidelines [24, 29]. Here, the developed guideline will be evaluated by the guideline developers, experts from the nominal group and two experts outside the nominal group. AGREE II has been translated and used by Iranian researchers before [30].

\section{Evaluating practicality of the guideline from service providers' perspective:}

To evaluate the practicality of the developed guideline, we will circulate it among at least 30 service providers asking them the last question in the AGREE II instrument to know if they recommend the usage of the guideline. We will also asked them if they have any suggestion to improve it. Subsequently, the comments and suggestions will be assessed by the research team and will be integrated in the final version of the guideline. 


\section{Discussion}

This study was designed with the aim of developing and validating a clinical guideline on the sexual and reproductive health of breast cancer survivors in Iran.

Same recommendations are not practical in different countries due to local, cultural and organizational differences. The development and validation of new guidelines also require substantial resources [28]. Therefore, here, we will use different techniques to avoid repeated work and save limited available resources while ensuring that the guideline meets the needs of Iranian breast cancer survivors. First, we will investigate current situation regarding services that survivors of breast cancer receive for their sexual and reproductive health in Iran. We will explore opinions of breast cancer survivors, their husbands, and service providers through a qualitative content analysis. Next, we will conduct a comprehensive literature review to find all available guidelines and recommendations in this regard worldwide. Then, we will use data from the qualitative study to modify currently available guidelines by adopting recommendation as they are or adapting them considering local needs also adding recommendations as required. The qualitative data will be collected from Tehran which is the city with the highest population in Iran and from referral centers having patients from around the country. Therefore, it will include views and experiences from a wide range of people. We will also use data from service providers at different levels and incorporate their ideas in the guideline to make it comprehensive and practical. The data will be collected from both public, semi-private and private sectors. Therefore, it will include people from a wide socioeconomic range. To validate the guideline, we will use AGREE II instrument and collect opinions of experts from outside the study group, policy makers and also future users in the field as well as the experts within the study group. This design will improve the feasibility and practicability of the guideline.

In this study, we will use an exploratory sequential mixed methods approach. Although this approach is more complex and includes more steps, it will lead to development of a guideline which is more valid and reliable. Mixed methods approach and multiple techniques have often been used by other groups to develop guidelines and protocols. Schünemann et al introduced GRADE-ADOLOPMENT approach in 2017 to develop new guidelines by combining three techniques (adopt, adapt and de-novo development of recommendations). They evaluated the recommendations using GRADE framework. However, they did not use qualitative data [18]. In 2020, Salarvand et al combined the results of a qualitative content analysis with these three techniques (adopt, adapt and de-novo development of recommendations) to develop a comprehensive clinical practice guideline for cancer therapy induced mucositis. They suggest this method as a practical approach to develop comprehensive clinical guidelines in developing countries [31]. Mixed methods approaches are currently being used by a number of Iranian study groups to develop multiple guidelines (e.g. sexual and reproductive health of surrogate mothers [32], perinatal palliative care for women with fetal anomaly diagnosis [33], and improving the childbirth experiences of Iranian women [34]).

Service providers can use recommendations of this guideline at different levels in all centers providing services to breast cancer survivors including hospitals, clinics, health centers, private offices, and 
palliative care and support centers.

During the process of breast cancer diagnosis and treatment, patients are often overwhelmed by multiple physical, mental and social problems, and usually ignore their sexual and reproductive health issues. On the other hand, there are not enough experts in this field with sufficient knowledge and skills. Therefore, patients usually get little help in this regard. This guideline will provide a scientific and practical framework for providing sexual and reproductive health services for survivors of breast cancer in Iran, and can significantly increase patients' quality of life. It can also be used as a model to develop guidelines for the sexual and reproductive health of survivors of the other kinds of cancer.

\section{Abbreviations}

ACS: American Cancer Society

AGREE II: Appraisal of Guidelines, Research and Evaluation II

AHRQ: Agency for Healthcare Research and Quality

ASCO: American Society of Clinical Oncology

BCY4: Breast Cancer in Young Women

CCO: Cancer Care Ontario

ESMO: European society for medical oncologists

ESO: European School of Oncology

GRADE: Grading of Recommendations, Assessment, Development and Evaluation

NCCN: National Comprehensive Cancer Network

NGT: Nominal group technique

NICE: National Institute for Clinical Excellence

PIPOH: Population concerned, Interventions of interest, target Professionals, expected Outcome, and Health care setting and context

SIGN: Scottish Intercollegiate Guidelines Network

\section{Declarations}

\section{Acknowledgements}


This paper is part of SR's PhD thesis on sexual and reproductive health. The authors would like to thank Tarbiat Modares University for supporting this research.

\section{Authors' contributions}

All authors were involved in the conception and design of the study. SR drafted the manuscript. All authors read the draft and provided input. ML and SAA provided key information and contributed to the writing process. SH and EM were the study advisors, and provided background information and helped with the methodology. EM helped with the qualitative study design. ML will supervise the research process. All authors have read and approved the final version of the manuscript.

\section{Funding}

This research is funded by Tarbiat Modares University, Tehran, Iran (Grant no. 78431).

\section{Availability of data and materials}

The developed guideline will be available from the corresponding author on request.

\section{Ethics approval and consent to participate}

Ethical approval for this study has been obtained from the ethics committee of Tarbiat Modares University, Tehran, Iran (IR.MODARES.REC.1397.207).

Written informed consent will be taken from each participant.

\section{Consent for publication}

Not applicable.

\section{Competing interests}

The authors declare that they have no competing interests.

\section{References}

1. Ferlay J, Colombet M, Soerjomataram I, Mathers C, Parkin DM, Piñeros M, et al. Estimating the global cancer incidence and mortality in 2018: GLOBOCAN sources and methods. Int J Cancer. 2019;144(8):1941-53. https://doi.org/10.1002/ijc.31937.

2. Ministry of Health and Medical Education. National cancer registry report. 2015. https://sghc.iums.ac.ir/files/sghc/files/pdf/Gozaresh_Keshvari_Sabte_Saratan_1394_-_Sent_(1).pdf. Accessed 10 Sep 2020.

3. Akbari ME, Sayad S, Sayad S, Khayamzadeh M, Shojaee L, Shormeji Z, et al. Breast Cancer Status in Iran: Statistical Analysis of 3010 Cases between 1998 and 2014. Int J Breast Cancer. 
2017;2017:2481021. https://doi.org/10.1155/2017/2481021.

4. American Cancer Society. Cancer Treatment \& Survivorship Facts \& Figures 2019-2021. 2019. https://www.cancer.org/content/dam/cancer-org/research/cancer-facts-and-statistics/cancertreatment-and-survivorship-facts-and-figures/cancer-treatment-and-survivorship-facts-and-figures2019-2021.pdf. Accessed 10 Sep 2020.

5. Nafissi N, Khayamzadeh M, Zeinali Z, Pazooki D, Hosseini M, Akbari ME. Epidemiology and Histopathology of Breast Cancer in Iran versus Other Middle Eastern Countries. MEJC. 2018;9(3):24351. https://doi.org/10.30476/mejc.2018.42130.

6. Ljungman L, Ahlgren J, Petersson LM, Flynn KE, Weinfurt K, Gorman JR, et al. Sexual dysfunction and reproductive concerns in young women with breast cancer: Type, prevalence, and predictors of problems. Psychooncology. 2018;27(12):2770-7. https://doi.org/10.1002/pon.4886.

7. Streb J, Jabłoński MJ, Słowik A, Babczyk D, Jach R. Indications for sexology consultation in women after surgical treatment due to breast cancer. Ann Agric Environ Med. 2019;26(2):379-84. https://doi.org/10.26444/aaem/89733.

8. Shahid Sales S, Hasanzadeh M, Sania Saggade S, Al Davoud SA. Comparison of sexual dysfunction in women with breast cancer: case control study. Tehran Univ Med J. 2017;75(5):350-7.

9. Harirchi I, Montazeri A, Zamani Bidokhti F, Mamishi N, Zendehdel K. Sexual function in breast cancer patients: a prospective study from Iran. J Exp Clin Cancer Res. 2012;31(1):20. https://doi.org/10.1186/1756-9966-31-20.

10. Bober SL, Reese JB, Barbera L, Bradford A, Carpenter KM, Goldfarb S, et al. How to ask and what to do: a guide for clinical inquiry and intervention regarding female sexual health after cancer. Curr Opin Support Palliat Care. 2016;10(1):44-54. https://doi.org/10.1097/spc.0000000000000186.

11. Carter J, Lacchetti C, Andersen BL, Barton DL, Bolte S, Damast S, et al. Interventions to Address Sexual Problems in People With Cancer: American Society of Clinical Oncology Clinical Practice Guideline Adaptation of Cancer Care Ontario Guideline. J Clin Oncol. 2018;36(5):492-511. https://doi.org/10.1200/jco.2017.75.8995.

12. Christian N, Gemignani ML. Issues with Fertility in Young Women with Breast Cancer. Curr Oncol Rep. 2019;21(7):58. https://doi.org/10.1007/s11912-019-0812-4.

13. Canada AL, Schover LR. The psychosocial impact of interrupted childbearing in long-term female cancer survivors. Psychooncology. 2012;21(2):134-43. https://doi.org/10.1002/pon.1875.

14. Ussher JM, Parton C, Perz J. Need for information, honesty and respect: patient perspectives on health care professionals communication about cancer and fertility. Reprod Health. 2018;15(1):2. https://doi.org/10.1186/s12978-017-0441-z.

15. Paluch-Shimon S, Cardoso F, Partridge AH, Abulkhair O, Azim HA, Jr., Bianchi-Micheli G, et al. ESOESMO 4th International Consensus Guidelines for Breast Cancer in Young Women (BCY4). Ann Oncol. 2020 https://doi.org/10.1016/j.annonc.2020.03.284.

16. Mody SK, Panelli DM, Hulugalle A, Su HI, Gorman JR. Contraception concerns, utilization and counseling needs of women with a history of breast cancer: a qualitative study. Int J Womens 
Health. 2017;9:507-12. https://doi.org/10.2147/ijwh.s136120.

17. Institute of Medicine (US) Committee on Standards for Developing Trustworthy Clinical Practice Guidelines; Editors: Robin Graham, Michelle Mancher, Dianne Miller Wolman, Sheldon Greenfield, and Earl Steinberg. Clinical Practice Guidelines We Can Trust. Washington (DC): National Academies Press (US); 2011.

18. Schünemann HJ, Wiercioch W, Brozek J, Etxeandia-lkobaltzeta I, Mustafa RA, Manja V, et al. GRADE Evidence to Decision (EtD) frameworks for adoption, adaptation, and de novo development of trustworthy recommendations: GRADE-ADOLOPMENT. J Clin Epidemiol. 2017;81:101-10. https://doi.org/10.1016/j.jclinepi.2016.09.009.

19. Runowicz CD, Leach CR, Henry NL, Henry KS, Mackey HT, Cowens-Alvarado RL, et al. American Cancer Society/American Society of Clinical Oncology Breast Cancer Survivorship Care Guideline. CA Cancer J Clin. 2016;66(1):43-73. https://doi.org/10.3322/caac.21319.

20. Barbera L, Zwaal C, Elterman D, McPherson K, Wolfman W, Katz A, et al. Interventions to address sexual problems in people with cancer. Curr Oncol. 2017;24(3):192-200. https://doi.org/10.3747/co.24.3583.

21. Melisko ME, Narus JB. Sexual Function in Cancer Survivors: Updates to the NCCN Guidelines for Survivorship. J Natl Compr Canc Netw. 2016;14(5 Suppl):685-9. https://doi.org/10.6004/jnccn.2016.0193.

22. Oktay K, Harvey BE, Partridge AH, Quinn GP, Reinecke J, Taylor HS, et al. Fertility Preservation in Patients With Cancer: ASCO Clinical Practice Guideline Update. J Clin Oncol. 2018;36(19):1994-2001. https://doi.org/10.1200/jco.2018.78.1914.

23. Murphy MK, Black NA, Lamping DL, McKee CM, Sanderson CF, Askham J, et al. Consensus development methods, and their use in clinical guideline development. Health Technol Assess. 1998;2(3):i-iv, 1-88.

24. Brouwers MC, Kho ME, Browman GP, Burgers JS, Cluzeau F, Feder G, et al. AGREE II: advancing guideline development, reporting and evaluation in health care. Cmaj 2010;182(18):E839-E42. https://doi.org/10.1503/cmaj.090449.

25. Graneheim UH, Lundman B. Qualitative content analysis in nursing research: concepts, procedures and measures to achieve trustworthiness. Nurse Educ Today. 2004;24(2):105-12. https://doi.org/10.1016/j.nedt.2003.10.001.

26. Polit DF, Beck CT. Essentials of Nursing Research: Appraising Evidence for Nursing Practice. 9th ed. Philadelphia: Wolters Kluwer; 2018.

27. Guyatt GH, Oxman AD, Vist GE, Kunz R, Falck-Ytter Y, Alonso-Coello P, et al. GRADE: an emerging consensus on rating quality of evidence and strength of recommendations. BMJ. 2008;336(7650):924-6. https://doi.org/10.1136/bmj.39489.470347.AD.

28. The ADAPTE Collaboration. The ADAPTE Process: Resource Toolkit for Guideline Adaptation. Version 2.0. 2009. http://www.g-i-n.net. Accessed 10 Sep 2020. 
29. AGREE Next Steps Consortium. The AGREE II Instrument [Electronic version]. 2017. http://www.agreetrust.org. Accessed 10 Sep 2020.

30. Ghanbari A, Rahmatpour P, Jafaraghaee F, Kazemnejad E, Khalili M. Quality assessment of diabetic foot ulcer clinical practice guidelines. J Evid Based Med. 2018;11(3):200-7. https://doi.org/10.1111/jebm.12304.

31. Salarvand S, Hemati S, Adibi P, Taleghani F, Saleki M. An Innovative Approach to Clinical Practice Guideline Adaptation in the Nursing Profession in a Developing Country. Cancer Manag Res. 2020;12:2255-64. https://doi.org/10.2147/cmar.s233542.

32. Goli M, Farajzadegan Z, Heidari Z, Kohan S. Reproductive and sexual Health of surrogate mothers, developing a care program: a protocol for mixed methods study. Reprod Health. 2019;16(1):23. https://doi.org/10.1186/s12978-019-0687-8.

33. Raisi Dehkordi Z, Kohan S, Rassouli M, Zarean E, Malekian A. Developing a perinatal palliative care service package for women with fetal anomaly diagnosis: protocol for mixed methods study. Reprod Health. 2020;17(1):32. https://doi.org/10.1186/s12978-020-0881-8.

34. Ghanbari-Homayi S, Fardiazar Z, Mohammad-Alizadeh-Charandabi S, Asghari Jafarabadi M, Mohamadi E, Meedya S, et al. Developing of a new guideline for improving birth experiences among Iranian women: a mixed method study protocol. Reprod Health. 2020;17(1):17. https://doi.org/10.1186/s12978-020-0868-5. 